\title{
A PORTABLE PULSED NEUTRON GENERATOR
}

\author{
A. SKOULAKIS ${ }^{1,2}$, G. C. ANDROULAKIS ${ }^{1,2}$, E. L. CLARK ${ }^{1}$, S. M. HASSAN ${ }^{1,5}$, P. LEE $^{6}$, J. CHATZAKIS ${ }^{1,2}$, \\ M. BAKAREZOS ${ }^{1,3}$, V. DIMITRIOU ${ }^{1,4}$, C. PETRIDIS $^{1,2}$, N. A. PAPADOGIANNIS ${ }^{1,3}$, M. TATARAKIS $^{1,2}$ \\ ${ }^{I}$ Center for Plasma Physics and Lasers, Technological Educational Institute of Crete (TEI) \\ 73133 Chania, 74100 Rethymno, Crete, Greece \\ m.tatarakis@chania.teicrete.gr \\ ${ }^{2}$ Department of Electronics, Technological Educational Institute of Crete (TEI), Chania, Greece \\ ${ }^{3}$ Department of Music Technology \& Acoustics, Technological Educational Institute of Crete (TEI) \\ 74100, Rethymnon, Greece \\ ${ }^{4}$ Department of Natural Resources \& Environment, Technological Educational Institute of Crete (TEI) \\ 73133, Chania, Greece \\ ${ }^{5}$ School of Nuclear Engineering, Purdue University, West Lafayette, IN 47907, USA \\ ${ }^{6}$ Natural Sciences and Science Education, National Institute of Education, Nanyang Technological University \\ Singapore 637616, Singapore
}

Published 25 February 2014

\begin{abstract}
The design and construction of a pulsed plasma focus device to be used as a portable neutron source for material analysis such as explosive detection using gamma spectroscopy is presented. The device is capable of operating at a repetitive rate of a few Hz. When deuterium gas is used, up to $10^{5}$ neutrons per shot are expected to be produced with a temporal pulse width of a few tens of nanoseconds. The pulsed operation of the device and its portable size are its main advantage in comparison with the existing continuous neutron sources. Parts of the device include the electrical charging unit, the capacitor bank, the spark switch (spark gap), the trigger unit and the vacuumfuel chamber / anode-cathode. Numerical simulations are used for the simulation of the electrical characteristics of the device including the scaling of the capacitor bank energies with total current, the pinch current, and the scaling of neutron yields with energies and currents. The MCNPX code is used to simulate the moderation of the produced neutrons in a simplified geometry and subsequently, the interaction of thermal neutrons with a test target and the corresponding prompt $\gamma$-ray generation.
\end{abstract}

Keywords: Pulsed power plasma devices; neutron sources; plasma dynamics; plasma sources.

\section{Introduction}

A dense plasma focus $(\mathrm{DPF})^{1}$ is a plasma accelerator consisting of two electrodes in a coaxial configuration. The inner electrode is usually the anode; its one end is connected

This is an Open Access article published by World Scientific Publishing Company. It is distributed under the terms of the Creative Commons Attribution 3.0 (CC-BY) License. Further distribution of this work is permitted, provided the original work is properly cited. 
with the cathode via an insulator sleeve that covers part of the anode's lower part, while the other end is kept open. Depending on the purpose of the experiment, the empty space in between the electrodes is filled with a certain gas. The DPF is connected to a capacitor bank whose electrostatic energy is transferred rapidly to the gas via a fast switch. A current sheath forms at the base of the DPF, which moves upwards due to a $\mathbf{J} \times \mathbf{B}$ force. When the sheath reaches the open end of the anode, it implodes radially, producing a short-lived column of hot dense plasma, which is finally disrupted by MHD instabilities. For systematic reasons, the time evolution of the phenomenon is divided into three successive stages: the dielectric breakdown of the gas, the axial phase and finally the radial phase, during which the focusing of the plasma into a miniature z-pinch occurs. Being able to produce hot dense plasma, a DPF offers the possibility to investigate plasma dynamics for a broad range of applications. ${ }^{2}$ Moreover, DPFs with appropriate filling gases may constitute excellent sources of X-rays, EUV and beams of energetic particles such as protons, neutrons, ions and electrons.

A DPF is usually characterized by the energy that can be stored in the capacitor bank and the peak current between the electrodes. Historically, the trend was to construct and operate large DPFs, with energies ranging from kJ to MJ, aimed mainly at massive radiation production. Their large size, however, poses certain problems such as nonportability, considerable cost and manpower needed for operation, and low repetition rate, if any. These, along with the plasma scalability, have led to the development of smaller DPF devices with energies of tens of joules, the so-called miniature DPFs. It is interesting to note that extensive research has proved that there are certain plasma parameters whose value remains within a narrow interval over a wide range of DPF energies: the electron density $\left(\sim 10^{24}\right.$ to $\left.10^{26} \mathrm{~m}^{-3}\right)$, the electron temperature ( $200 \mathrm{eV}$ to 2 $\mathrm{keV})$, the plasma temperature $(\sim 1 \mathrm{keV})$ and the axial/radial velocity $\left(\sim 10^{5} \mathrm{~m} / \mathrm{s}\right)$. As we shall see below, these plasma parameters are relevant to certain DPF parameters, thus providing a useful tool for designing smaller devices with performance comparable to their larger counterparts.

An interesting aspect of the DPF is that when deuterium is used as a filling gas, fast neutrons with energy distribution well centered around $2.45 \mathrm{MeV}$ are produced. The neutron emission has a $4 \pi$ distribution, and its duration is of the order of tens of nanoseconds. In the past, it was thought that these neutrons were of purely thermonuclear origin, that is, thermal collisions between deuterons in the plasma bulk, resulting in isotropic neutron radiance. However, simultaneous measurements of the neutron yield $\left(Y_{\mathrm{n}}\right)$ along the axial $\left(0^{\circ}\right)$ and the radial $\left(90^{\circ}\right)$ directions has revealed an anisotropy $Y_{\mathrm{n}}\left(0^{\circ}\right)$ / $Y_{\mathrm{n}}\left(90^{\circ}\right)$ of $\sim 1.2$ to 3 , ruling out the possibility of a pure thermonuclear mechanism. Moreover, the average energy of neutrons in the axial direction was found to be greater than the energy of thermonuclear neutrons $(2.45 \mathrm{MeV})$, providing more evidence that there is a non-thermal component contributing to the total neutron yield. ${ }^{3}$ Later, it was proposed that the non-thermal mechanism is the so-called beam-target effect, according to which accelerated deuterons collide with thermal deuterons and neutral atoms. To date, it is widely accepted that both thermonuclear and beam-target effects contribute, but the 
scaling of their relative strength with the capacitor energy as well as the details of the ions acceleration mechanism are still under debate.

Our goal is to construct a tabletop DPF $(\sim 100 \mathrm{~J})$ that will serve as a pulsed neutron source. The latter will subsequently be used for identifying organic substances via gamma spectroscopy and in particular, to detect explosives. The desirable characteristics are mainly portability and a decent repetition rate in order to compensate for the necessarily low neutron yield that a miniature DPF has. The main advantage of a DPF neutron source over continuous sources with similar spectrum such as americiumberyllium (AmBe) or ${ }^{252} \mathrm{Cf}$ is that it is of the on/off type; consequently, it is easier to handle and store. Moreover, the short temporal duration of the neutron burst allows time resolved activation analysis whenever this is necessary.

Neutron activation analysis (NAA) $)^{4,5}$ is a well-known method for non-destructive determination of the concentration of chemical elements. By focusing solely on the nuclei of atoms, it allows discrete sampling of elements no matter the chemical structure of the irradiated sample. The principle of NAA is rather simple. A test substance is bombarded with neutrons of certain energy; non elastic collisions between thermal neutrons and nuclei result in unstable compound nuclei via neutron capture. The latter de-excite spontaneously into stable configurations accompanied by emission of one or more prompt gamma photons. The resulting nuclei are in most cases radioactive, which in turn decay into other particles and delayed gamma photons. By analyzing the spectrum of prompt or delayed gamma rays and after comparing with tabulated nuclear data for gamma ray emission, one may trace back the elements of which the test sample consists. Fast neutron activation analysis is also a possibility, requiring, however, considerably more energetic neutrons than a deuterium filled DPF can produce.

\section{Design and Construction}

To design a DPF device, one usually starts by determining the energy stored in the capacitor bank that will eventually be transferred to the fuel inside the chamber. Our capacitor bank consists of four identical capacitors $(C=200 \mathrm{nF}, V=30 \mathrm{kV}, L=15 \mathrm{nH})$ yielding a total capacitance of $0.8 \mu \mathrm{F}$. Depending on their charging voltage, the capacitor bank may provide electrostatic energy ranging from 100 to $160 \mathrm{~J}$. The electric circuit diagram of the assembly is depicted in Fig. 1. A high voltage power supply charges the capacitors up to the desired voltage. To trigger the discharge, we use an in-house designed trigger unit that produces a $2.5 \mathrm{kV}, 1 \mu$ s impulse that is then transmitted to the pseudo spark switch (PSS) via a pulse shaping circuit. ${ }^{6}$ The load of the capacitors is finally carried to the fuel through 16 coaxial lines in order to reduce the total resistance.

The PSS unit is a TDI1-150k/25 type (copper arc thyratron) capable of producing currents well above a hundred kA. Moreover, it has a lifetime of $10^{7}$ shots (for E $100 \mathrm{~J}$ ), and therefore, it can operate continuously at a few Hertz for several weeks without undergoing repairs. Yet another advantage of the TDI1-150k/25 type PSS is its reliability of firing practically in $100 \%$ of cases with a jitter time less than $4 \mathrm{~ns}$. 


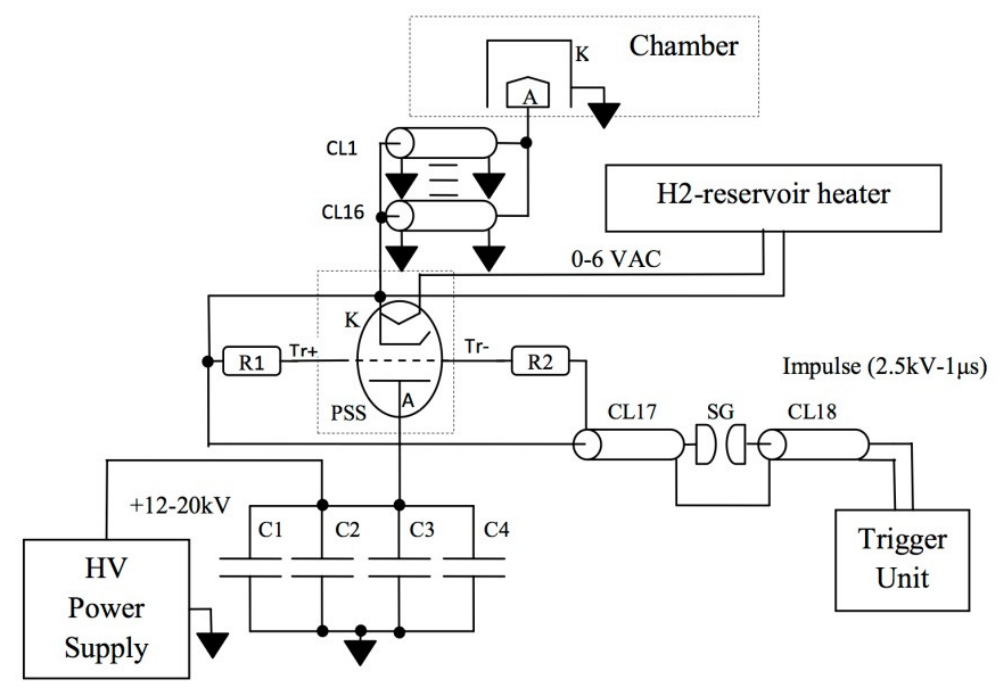

Fig.1. The electric circuit.

The assembly is capable of operating at a few $\mathrm{Hz}$ without cooling and producing a peak discharge current of $\sim 45-65 \mathrm{kA}$ (measured by a Rogowski coil) with a quarter cycle of $260 \mathrm{~ns}$. A key aspect for constructing a fast DPF is to keep inductance as low as possible. For small devices, this strongly depends on the connections, and therefore, components must be connected in compact configuration. The external inductance of the assembly, including all electric components and the chamber, is slightly less than $50 \mathrm{nH}$.

As already mentioned above, a remarkable property of a DPF is that there are certain design parameters that are practically constant over a wide range of energies. Consider the so-called "drive parameter" $=I_{0} / \alpha \sqrt{p_{0}}$, with $I_{0}$ being the peak current, $\alpha$ the anode radius and $p_{0}$ the optimum ambient gas pressure for neutron generation. The parameter $S$ is the designing factor that really determines how fast the shock wave propagates, that is, the velocity of the current sheath in both axial and radial phases. Consequently, it is also related to the temperature of the final pinch plasma upon which many radiation quantities are dependent. The numerical value of $S$ is $77 \mathrm{kA} \cdot \mathrm{cm}^{-1} \cdot \mathrm{mbar}^{1 / 2}$, with variance less than $10 \%$ for facilities ranging from a few tens of joules to MJ. For a given DPF, the drive parameter is useful for determining the gas pressure that optimizes the neutron yield.

One more important parameter is the plasma energy density ${ }^{7} E_{p}=E / V_{p}$, where $E$ is the energy of the capacitor bank and $V_{p}$ is the volume of the pinch plasma column. It may be observed that only a fraction of $E$ is finally deposited in the pinch plasma. However, the parameter $E_{p}$ has been shown experimentally to be of the order of $10^{10} \mathrm{~J} \cdot \mathrm{m}^{-3}$. As observed by Lee and Serban, ${ }^{8}$ the dimensions of the plasma column are proportional to the anode radius. According to their scalling laws, the final pinch radius is $r_{\min } \sim 0.12 \alpha$, and the maximum pinch length is $z_{\max } \sim 0.8 \alpha$. Therefore, $V_{p}=\pi \times(0.12 \alpha)^{2} \times 0.8 \alpha=$ $0.036 \alpha^{3}$, and thus the plasma energy density may be expressed as a function of the anode radius solely: $E_{p}=28 E \alpha^{-3}$. 
For designing a miniature DPF with $E \sim 100 \mathrm{~J}$, the value $E_{p}=5 \times 10^{10} \mathrm{~J} \cdot \mathrm{m}^{-3}$ is expected, and therefore, the anode radius must be $\alpha \sim 4 \mathrm{~mm}$. The anode length $z_{a}$ may be estimated as follows. The optimum case would be the peak current, occuring at the quarter of the cycle, to coincide with the pinch, i.e., $a / v_{r}+z_{a} / v_{a}=T / 4$, where $v_{r}$ and $v_{a}$ are the radial and the axial velocities respectively. Given that $v_{r}=2.5 \times 10^{5} \mathrm{~m} / \mathrm{s}, v_{a}=10^{5} \mathrm{~m} / \mathrm{s}$, and $T \sim 1.1 \mu \mathrm{s}$, it follows that $z_{a}=23 \mathrm{~mm}$. Moreover, we chose the tapered geometry towards the open end, as it has been observed that this shape results in a faster current sheath and enhances neutron production. ${ }^{9,10}$

The role of the insulator is not as innocent as it may seem. Its length severely affects the initial gas breakdown and consequently, both subsequent phases. Although the formation of the initial current sheath strongly depends on the pressure of the fuel, it has been found that for optimum pressure, the insulator length determines the curvature of the current sheath, and further, it must be such that a uniform and azimuthally symmetric current sheath appears. Any deviations from the optimum length result in spokes in the current sheath, which in turn decreases the neutron yield. Quantitavely, this is encoded in the parameter $L_{\text {ins }} / b$, with $L_{\text {ins }}$ being the length of the insulator and $b$ the inner radius of the cathode. ${ }^{11}$ The more this parameter deviates from unity, the lower the neutron production is. The material of the insulator is also important. Experimentally, it appears that neutron production increases linearly with the product $p \times \varepsilon_{\mathrm{r}}$, where $p$ is the fuel pressure and $\varepsilon_{\mathrm{r}}$ is the dielectric constant of the insulator material. ${ }^{12}$ Therefore, we chose to use Pyrex for having large $\varepsilon_{\mathrm{r}}(\sim 4.5-6.0)$, being cost effective and easy to modify.

\section{MCNPX Simulation}

In the literature, it is possible to find many scaling laws relating neutron yield with the peak (or the pinch) current. All of them seem to agree that the scaling has the general form $Y_{\mathrm{n}} \propto I_{\text {peak }}^{k}$ for some power $k$, usually between two and four. According to Lee's model, ${ }^{13} k=3.9$ and the proportionality factor is $9 \times 10^{10}$, which in our case gives $Y_{\mathrm{n}}=10^{5}$ neutrons per shot. Lee's model successfully predicted the current of our device, which suggests that the neutron yield won't be far from the aforementioned value.

As already mentioned, the neutrons that are produced by a deuterium filled DPF are not fast enough for fast neutron activation analysis. Primarily we are interested in explosives detection, which can be done by identifying their relative concentrations of $\mathrm{C}$, $\mathrm{O}, \mathrm{N}$ and $\mathrm{H}$. Their cross sections for neutron capture ${ }^{14}$ are large only in the region of subthermal energies, and therefore, thermalization of neutrons is necessary.

We employ the MCNPX code ${ }^{15}$ in a simple geometry in order to maximize the thermal neutrons population with respect to the moderator's size and to obtain qualitative information of the expected gamma spectrum so as to aid procurement of a gamma spectroscopy system. The scenario (depicted in Fig. 2) that has been tested is the following: A $2 \mathrm{~cm}$ collimated beam of $2.45 \mathrm{MeV}$ neutrons is incident onto a $4 \mathrm{~cm} \times 4 \mathrm{~cm}$ polyurethane moderator of particular thickness. A $2 \mathrm{~cm} \times 4 \mathrm{~cm} \times 4 \mathrm{~cm}$ block of TNT is placed behind the moderator as a test target. TNT $(\rho=1.654)$ is modeled with the following atomic fractions: C: $0.35, \mathrm{H}: 0.25, \mathrm{~N}: 0.15$ and $\mathrm{O}: 0.25$. 


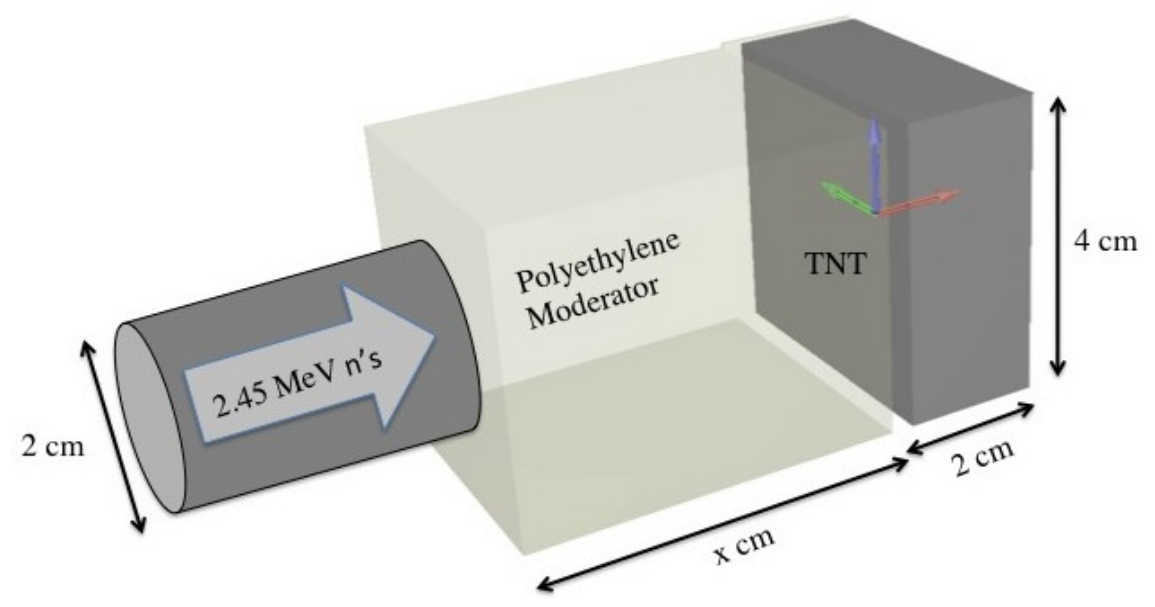

Fig. 2. MCNPX geometry.

The outputs of the code are (i) the neutron spectrum at the end of the moderator and (ii) the prompt gamma spectrum at the side face of the TNT block. It should be noted, however, that the geometry above is not realistic, as a complete simulation of the plasma focus, moderator, detector and shielding is required. In particular, the photon count surface picks up carbon and hydrogen gamma emission from the moderator, and moreover, actual detector measurements will be modified by characteristics of the NaI scintillator detector system. In addition, the actual distribution of neutrons, the real distances between the source, moderator, target and detectors, and the gamma fluence of the moderator itself must be taken into account.

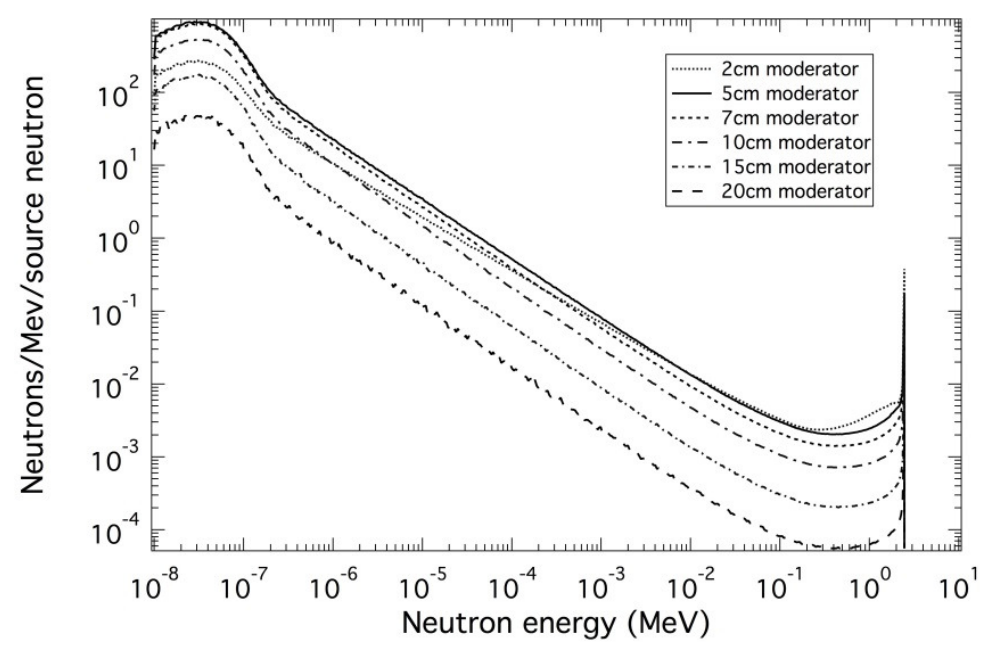

Fig. 3. Thermal neutrons per MeV per source neutron as a function of thermal neutron energy. 
The moderated neutrons per $\mathrm{MeV}$ and per source fast neutron are plotted in Fig. 3 as a function of energy for various moderator thicknesses. It is seen that the optimum thickness is $5 \mathrm{~cm}$, yielding the greatest number of sub-thermal neutrons.

Figure 4 shows the prompt gamma spectra using $2 \mathrm{~cm}$ and $5 \mathrm{~cm}$ thick moderators, respectively. It is seen that the peaks with the $5 \mathrm{~cm}$ moderator are an order of magnitude higher. The lower peaks in the spectrum have poor statistics associated with them in the MCNPX calculation, so their relative magnitudes compared to the larger peaks contain no quantitative information. In a real measurement, these peaks would be difficult to identify, and the gamma spectroscopy would be confined to studying the relative ratios of the dominant peaks.
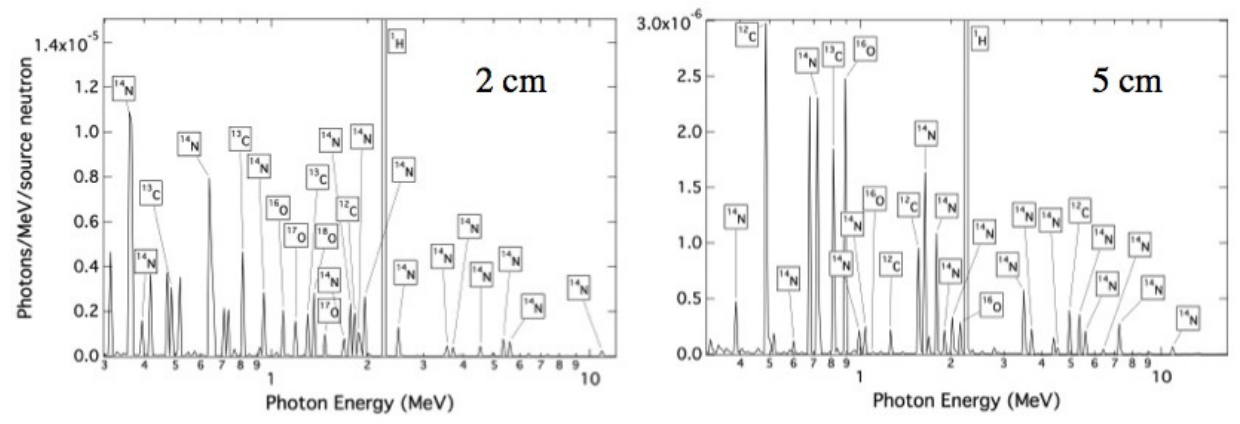

Fig. 4. Prompt $\gamma$ spectrum for $2 \mathrm{~cm}$ and $5 \mathrm{~cm}$ moderators.

\section{Conclusion}

A miniature DPF has been designed and constructed in order to serve as a portable neutron source for prompt gamma spectroscopy analysis. The Lee model has been used to simulate the assembly's electric response. The predicted peak current and quarter cycle are in good agreement with the experimental ones. According to the same model, we expect to produce $10^{5}$ neutrons per shot. As a deuterium filled DPF produces fast neutrons, it is necessary to moderate them to thermal energies. The MCNPX code has been used to arrive at conclusions about the moderator's thickness, as well as the number of shots required for explosives identification. We anticipate that, given the moderate yield of the neutron source, multiple shots will be necessary to obtain an adequate neutron spectrum.

\section{Acknowledgements}

This research has been co-financed by the European Union (European Social Fund ESF) and Greek national funds through the Operational Program "Education and Lifelong Learning" of the National Strategic Reference Framework (NSRF) - Research Funding Program: ARCHIMEDES III. Investing in knowledge society through the European Social Fund (action 16, Proposal Acronym: NEUTRON SOURCE). 


\section{References}

1. J. W. Mather, Phys. Fluids 8, 366 (1964).

2. V. A. Gribkov, Current and perspective applications of dense plasma focus devices, in Proc. $17^{\text {th }}$ IAEA Technical Meeting on Research Using Small Fusion Devices, AIP Conference Proceedings 996 (2008), p. 51, doi:http://dx.doi.org/10.1063/1.2917031.

3. F. Castillo et al., J. Phys. D: Appl. Phys. 33, 141 (2000).

4. G. Vourvopoulos and P. C. Womble, TALANTA 54, 459 (2001).

5. A. Buffler, Rad. Phys. and Chem. 71, 853 (2004).

6. J. Chatzakis et al., Rev. Sci. Instrum. 79, 086103 (2008).

7. L. Soto, Plasma Phys. Control. Fusion 47, A361 (2005).

8. S. Lee and A. Serban, IEEE Trans. Plasma Sci. 24, 1101 (1996).

9. R. K. Rout, A. Shyam and V. Chitra, Ann. Nucl. Energy 18, 357 (1991).

10. M Zakaullah, Imtiaz Ahmad, A. Omar, G. Murtaza and M. Beg, Plasma Sources Science Technology 5, 544 (1996).

11. H. R. Yousefi, G. R. Etaati and M. Ghorannevis, in Proc. 31st EPS Conference on Plasma Phys., ECA 28G (2004), P-5.006, http://epsppd.epfl.ch/London/pdf/P5_006.pd.

12. F. N. Beg, M. Zakaullah and G. Murtaza, Physica Scripta 46, 152 (1992).

13. S. Lee and S. H. Saw, Journal of Fusion Energy 27, 292 (2008).

14. A. P. Barzilov, Ivan S. Novikov and Phillip C. Womble, Material Analysis Using Characteristic Gamma Rays Induced by Neutrons, in Gamma Radiation, ed. Prof. Feriz Adrovic (InTech Open Access, 2012), p. 17, ISBN: 978-953-51-0316-5.

15. J. S. Hendricks et al., MCNPX, Version 2.6.0, Los Alamos National Laboratory Report LAUR-06-7991 (2008). 